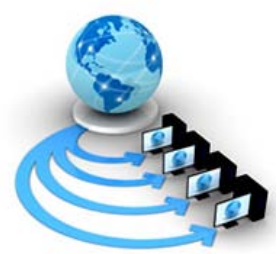

Volume 8, No. 7, July - August 2017

International Journal of Advanced Research in Computer Science

RESEARCH PAPER

Available Online at www.ijarcs.info

\title{
WEB PAGE ANTICIPATION SYSTEM USING MARKOV MODEL
}

\author{
Shivangi Sorout \\ Department of computer science \& Application \\ Maharishi Dayanand University (M.D.U) \\ Rohtak, Haryana
}

\author{
Dr. Balkishan (Assistant Professor) \\ Department of computer science \& Application \\ Maharishi Dayanand University (M.D.U) \\ Rohtak Haryana
}

\begin{abstract}
In the era of digital world, Information can be accessed with the help of Internet on the World Web. Due to the enormous growth of digital information, the limited bandwidth of the network is not utilized in an e fficient way. With the help of this "Web Page Anticipation System", we are trying to get rid of this problem. It provides us framework to incorporate the usage of pre-fetching mechanism, Clustering, Markov Model and Prediction Architecture. This framework allows us to anticipate the web page in advance with the help of user's currently accessed web page.
\end{abstract}

Keywords: com Web page anticipation, Pre-fetchimg Clustering, Markov model, Users sessions

\section{INTRODUCTION}

Owing $\mathrm{t}$ ot he e normous growth o fW orld W ide Web, congestion and overloading of server occurs. Due to problems incurred by the server in managing large information, latency of $\mathrm{c}$ ommunication channel is s ubstantially $\mathrm{r}$ educed. $\mathrm{V}$ arious techniques for latency reduction are web catching, pre-fetching and preopening. Need of this web page anticipation system is required in the er a of e-commerce digital w orld where ev ery transaction is depended only on the efficiency of how fast we are able to accesses the required web page within the particular time $\mathrm{s}$ lot. $\mathrm{R}$ esearchers us e different ki nd of $\mathrm{t}$ echniques comprising $\mathrm{M}$ arkov $\mathrm{M}$ odel $\mathrm{f}$ or ne $\mathrm{xt} \mathrm{w}$ eb pa ge anticipation, clustering and prediction A rchitecture. F or im plementing this web $\mathrm{p}$ age a nticipation $\mathrm{m}$ odel, na vigational be havior of th $\mathrm{e}$ current users is stored in the web log files.

After the $\mathrm{i}$ dentification of the navigational $\mathrm{b}$ ehavior o $\mathrm{f}$ current us ers, Clustering is performed. Clustering is the $\mathrm{m}$ ain ingredient us ed in the exploratory data mining and commonly used in the statistical data analysis. M ain task of clustering is to segregate the group of objects into different groups so that all objects in the one particular group are of similar nature. Scope of clustering is wide and u sed in the many fields like machine learning, image analysis, information retrieval, bioinformatics, data c ompression and c omputer $\mathrm{g}$ raphics. Clustering models used in the Clustering are connectivity models, centroid based model (k-means algorithm), distribution model expectation-maximization model) and density model.

When the Clusters are formed we have to use the prediction algorithm to predict the next possible states. After that Markov model is a pplied on the clustering s ets. M arkov m odel is a stochastic $\mathrm{m}$ odel $\mathrm{w}$ hich $\mathrm{i} \mathrm{su}$ sed f or the designing the continuously $\mathrm{c}$ hanging $\mathrm{s}$ ystem $\mathrm{i} \mathrm{n}$ va riable time $\mathrm{p}$ eriod a $\mathrm{t}$ different $t$ ime s lots. In th is model future $s$ tate is depend $n$ ot only on the current state but also on the previous states covered. In the Markov model we have to train the model by estimating the transition probability which is denoted by:

$$
\mathrm{Aij}=\mathrm{P}(\mathrm{Q}(\mathrm{t}+1)=\mathrm{Si} \mid \mathrm{Qt}=\mathrm{Sj})
$$

Where, Aij is the probability of going to the new state Si at time $\mathrm{t}+1$ from state $\mathrm{Sj}$ at time $\mathrm{t}$.

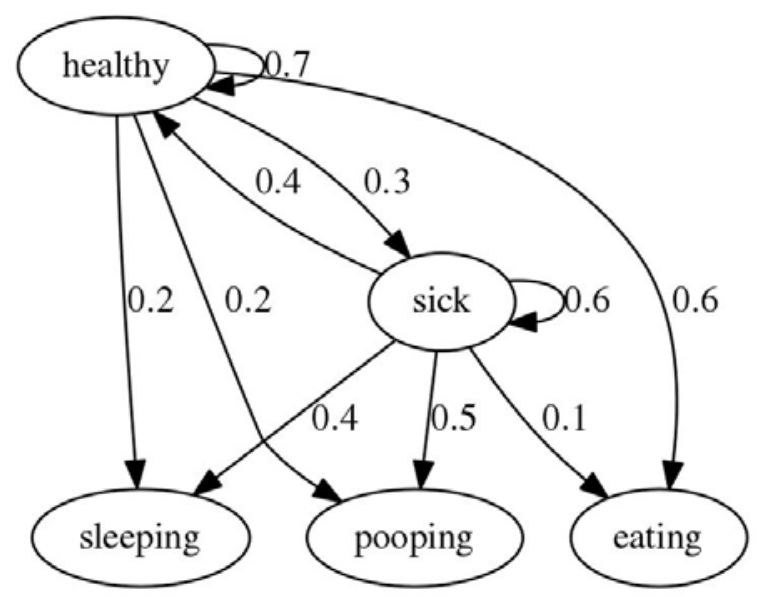

Figure1. Example: Markov Model States and their Transition Probability

The first order Markov model provides us a simple way of to accumulate sequential dependence, however it does not take the aspect of 1 ong $t$ erm $m$ emory web $n$ avigational behavior. Higher order M arkov m odel are $u$ seful fo $r t$ he prediction of navigational path. But with the increase of order of the Markov model subsequently there $w$ ill be exponential increase in the complexity of state space. In turn we require the huge a mount of $t$ raining $d$ ata. A $\mathrm{s} t$ he $\mathrm{n}$ umber of $\mathrm{s}$ tates increase, $\mathrm{s}$ ystems which $\mathrm{n}$ eeds to predict $\mathrm{f}$ ast $\mathrm{t}$ heir $\mathrm{p}$ rediction a ccuracy is plummeted to large extent. So the need of the hour is to have that kind of system which predict fast with enhanced accuracy.

\section{RELATED WORK}

The review of past investigation serves as a guide to the researchers as it av oids duplications $i \mathrm{n}$ the field. $\mathrm{T}$ he knowledge of $w$ hat $h$ as al ready $b$ een done $i \mathrm{n} t$ he a rea of investigation regarding the methods used for data is important. Research in any field implies a step ahead in exploration of the unknown. Any researcher to be able to take this step should be adequately prepared for it. One such preparation is gathering of 
knowledge of much has already been done in the given field. A step towards unkno wn $\mathrm{c}$ an only b e taken after the review of literature an $\mathrm{dr}$ esearches $\mathrm{d}$ one in that ar ea. Any research without $\mathrm{s}$ uch a review is li kely to be a b uilding $\mathrm{w}$ ithout foundation. Thus, $t$ he review o fr elated $l$ iterature $i \mathrm{~s}$ an indispensable step in research.

Yang et al. (2004) studied different a ssociation rule based methods for web request prediction. The association rules for web access prediction involve dealing with too many rules and it is not easy to find a suitable subset of rules to make accurate and reliable predictions. $F$ ive di fferent $r$ epresentations o $f$ association $\mathrm{r}$ ules are: $\mathrm{S}$ ubset rules, $\mathrm{S}$ ubsequence $\mathrm{r}$ ules, Latest subsequence rules, Substring rules a nd Latest substring rules. The au thor $\mathrm{c}$ oncerned the $\mathrm{p}$ recision of $\mathrm{t}$ hese five as sociation rules $r$ epresentations using $d$ ifferent selection methods, $t$ he latest substring rules were proven to have the highest precision with decreased number of rules [1].

Liu et al. (1998) introduced a customized marketing based on the web approach using a c ombination of $\mathrm{c}$ lustering a nd association rules. $\mathrm{T}$ he a uthor collected information a bout customers us ing forms, $\mathrm{W}$ eb s erver $\mathrm{log}$ files a nd cookies. It categorized c ustomers a ccording to the information c ollected. Since k-means clustering algorithm works only with numerical data, $t$ he authors us ed PAM ( Partitioning a round $M$ edoids) algorithm to cluster data using categorical scales. Then perform association rule techniques on each cluster [2].

Kim e t a 1. (2004) introduced c ombination of a $11 \mathrm{t}$ hree models $t$ ogether. It $\mathrm{i}$ mproves the performance of Markov model, sequential as sociation rules, as sociation $r$ ules a nd clustering $\mathrm{b}$ y combining a $1 \mathrm{t}$ hese $\mathrm{m}$ odels $\mathrm{t}$ ogether. For instance, Markov model is used first. If MM cannot cover an active session or a state, sequential association rules are used. If sequential as sociation rules cannot cover the state, as sociation rules a re used. I $\mathrm{f}$ association $\mathrm{r}$ ules $\mathrm{c}$ annot cover $\mathrm{t}$ he $\mathrm{s}$ tate, clustering algorithm is applied. The a uthor's w ork im proved recall and it did not improve the Web page prediction accuracy [3].

Vakali et a 1. ( 2003) categorized w eb data c lustering i nto two c lasses (I) u sers' s essions-based and (I I) link-based. The former uses the web log data and tries to group together a set of users' $\mathrm{n}$ avigation $\mathrm{s}$ essions $\mathrm{h}$ aving $\mathrm{s}$ imilar characteristics. I $\mathrm{n}$ web log data provide information about activities performed by $\mathrm{a} u$ ser $\mathrm{f}$ rom $\mathrm{t}$ he $\mathrm{m}$ oment $\mathrm{t}$ he $\mathrm{u}$ ser enters a $\mathrm{w}$ eb s ite to the moment the same user leaves it. The records of users' actions within a web site are stored in a log file. Each record in the log file co ntains the cl ient's IP ad dress, the $\mathrm{d}$ ate an $\mathrm{d}$ time the request is r eceived, the r equested obj ect and some a dditional information -such as protocol of request, size of the object etc [4].

Sarukkai et al. (2000) discovered e normous growth in the number of documents i $\mathrm{n}$ he W WW i ncreases $\mathrm{t}$ he $\mathrm{n}$ eed for improved link navigation and path analysis models. The author introduced lin $\mathrm{kp}$ rediction a nd $\mathrm{p}$ ath analysis are important problems with a w ide $r$ ange of applications ranging f rom personalization to web server request prediction. The sheer size of the WWW coupled with the va riation in us er's navigation patterns $\mathrm{m}$ akes $\mathrm{t}$ his a $\mathrm{v}$ ery $\mathrm{d}$ ifficult $\mathrm{s}$ equence $\mathrm{m}$ odeling problem. The author observed Markov chains allow the system to dynamically m odel the U RL acc ess $p$ atterns. The Markov chain $\mathrm{m}$ odel can a lso be used in a ge nerative $\mathrm{m}$ ode $\mathrm{t} \mathrm{o}$ automatically o btain tours. The Markov transition matrix $\mathrm{c}$ an be analyzed further using Eigen-vector decomposition to obtain personalized hubs/authorities. The utility of the Markov chain approach is demonstrated i $\mathrm{n} \mathrm{m}$ any d omains: H TTP request prediction, $\mathrm{s}$ ystem $\mathrm{d}$ riven adaptive $\mathrm{w}$ eb na vigation, $\mathrm{t}$ our generation, and detection of personalized hubs/authorities from user navigation profiles. The ge nerality and power of Markov chains is a $\mathrm{f}$ irst $\mathrm{s}$ tep $\mathrm{t}$ owards th e a pplication o $\mathrm{f} p$ owerful probabilistic models to $\mathrm{w}$ eb path a nalysis and lin $\mathrm{k}$ prediction [5].Khalil et al. (2007) introduced concept of prediction of the next page to be accessed by web users. This attracted a large amount of research w ork lately du e to the positive impact of such p rediction on different areas of $\mathrm{w}$ eb based a pplications. Major techniques applied for this intention a re Markov model and clustering. Low order Markov models are coupled with low accuracy, high order Markov models are associated with high state space complexity. On the other hand, clustering methods are unsupervised methods, and normally are not used for classification directly. It involves incorporating clustering with low order Markov model techniques. The pre-processed data is divided into m eaningful c lusters then the clusters are used as training da ta $\mathrm{w}$ hile pe rforming $2^{\text {nd }}$ order $\mathrm{M}$ arkov $\mathrm{m}$ odel techniques. Different distance measures of k-means clustering algorithm are examined in order to find a n optimal one. The author revealed that incorporating clustering of web documents according t o Web s ervices with 1 ow or der M arkov m odel improves the web page prediction accuracy [6].

Deshpande e t a 1. ( 2001) i ntroduced pr oblem of pr edicting a user's behavior on a web site has gained importance due to the rapid gr owth o ft he $\mathrm{W}$ orld $\mathrm{W}$ ide $\mathrm{Web}$ and the ne ed $\mathrm{t} o$ personalize an $\mathrm{d} i$ nfluences a $\mathrm{u}$ ser's browsing e xperience. Markov models and its variations found to be well suited for addressing $t$ his problem. $D$ ifferent va riations o $f$ Markov models, it found that higher-order Markov models display high predictive accuracies on web sessions that it can predict. However, higher-order models are also extremely complex due to their large number of states, which increases their space and run-time requirements. $T$ he author $p$ resented $d$ ifferent techniques for intelligently selecting $\mathrm{p}$ arts of $\mathrm{d}$ ifferent $\mathrm{o}$ rder Markov models so that the resulting model has a reduced state complexity, while maintaining a high predictive accuracy [7].

Zacharouli et al. (2009) introduced learning algorithms for web page $r$ ank pr ediction, linear $r$ egression models a nd combinations of $\mathrm{r}$ egression with pr obabilistic $\mathrm{c}$ lustering and Principal Components A nalysis (PCA). These models learned from time-series data sets and can predict the ranking of a set of web pages in some future time. The algorithm used separate linear re gression models. T his further e xtended by applying probabilistic clustering based on the EM algorithm. Clustering allows for the web pages to be grouped together by a mixture of regression m odels. A different $m$ ethod combined 1 inear regression with PCA so as dependencies between different web pages can be exploited. A 11 the methods e valuated us ing $\mathrm{r}$ eal data $s$ ets obtained $\mathrm{f}$ rom I nternet A rchive, Wikipedia a nd Yahoo! ranking lists. It also study the temporal robustness of the prediction framework. Overall the system constitutes a set of tools for high accuracy page rank prediction which can be used for efficient resource management by search engines [8].

Spiliopoulou et al. (1999) investigated w eb s ite de sign is currently based on interests of web site visitors and assumptions a bout their e xact $\mathrm{b}$ ehavior. Concrete knowledge on the $\mathrm{w}$ ay vi sitors $\mathrm{n}$ avigate $\mathrm{i} \mathrm{n}$ a $\mathrm{w}$ eb s ite could prevent disorientation a nd he lp ownersi $n$ placing $\mathrm{i}$ mportant information exactly where $t$ he vi sitors 1 ook $f$ or it. Web utilization miner tool can provide such knowledge. The general problem a ddressed is gi ven a num ber of $t$ raversed pa ths 
discovers s ub-paths w ith s tructural or s tatistical p roperties of interest. All nodes in a sub-path are of equal Importance. Subpaths having only some nodes in common be combined into a pattern that shows the desired properties as a whole to capture the ambiguous e xpressions of $t$ his $p$ roblem. $T$ he a uthor described a powerful mining language by which the expert can specify the d esired s tructural a nd s tatistical properties of the patterns. To efficiently discover paths which when c ombined result in such desirable patterns, an innovative technique based on the processing of ag gregated sequence several optimization steps are performed to further reduce the mining search space [9].

Mukhopadhyay e t a 1. ( 2011) s tudied about pre-fetching models based on $\mathrm{d}$ ecision $\mathrm{t}$ rees, $\mathrm{M}$ arkov chains, an $\mathrm{d} \mathrm{p}$ ath analysis. The author described increase uses of dynamic pages, frequent changes in site structure and user access patterns have limited th e e fficacy of these s tatic $t$ echniques. O ne of the techniques that are used for improving user latency is Caching and another is W eb pre-fetching. A pproaches that bank solely on caching offer limited performance improvement because it is $d$ ifficult $f$ or caching $t$ o ha ndle $t$ he large num ber o $f$ increasingly diverse files. An agent based method is proposed here to cluster related pages into different categories based on the access patterns. Additionally page ranking is us ed to build up the prediction model at the initial stages when users are yet to invoke any page [10].

Kumar et al. (2011) presented web provides a c orpus of design e xamples unpa ralleled i $\mathrm{n}$ hum an hi story. L everaging existing designs to produce new pages is difficult. The author introduced the Bricolage algorithm $\mathrm{f}$ or automatically transferring design and content between $\mathrm{Web}$ pages. Bricolage introduces a novel structured prediction technique that learns to create coherent mappings between pages by training on humangenerated exemplars. The produced mappings can then be used to au tomatically $t$ ransfer the content from o ne page i nto the style a nd layout of another. The author s hown that Bricolage can learn to accurately reproduce human page mappings, and that it provides a general, efficient, and automatic technique for retargeting content between a variety of real web pages [11].

Dutta et a l. (2011) studied web p age prediction p lays an important role by predicting and fetching probable web page of next request in advance, resulting in reducing the us er latency. The users surf the internet either by entering URL or search for some topic or through link of same topic. For searching and for link prediction, c lustering plays a $\mathrm{n}$ i mportant role. $\mathrm{W}$ eb page prediction model give us significant i mportance to the us er's interest us ing $t$ he $\mathrm{c}$ lustering $\mathrm{t}$ echnique and the na vigational behavior of $\mathrm{t}$ he us er through $\mathrm{M}$ arkov m odel. The $\mathrm{c}$ lustering technique i s us ed $\mathrm{f}$ or the a ccumulation of $\mathrm{t}$ he $\mathrm{s}$ imilar $\mathrm{w}$ eb pages. $\mathrm{S}$ imilar w eb pages of $\mathrm{s}$ ame $t$ ype $\mathrm{r}$ eside in the $\mathrm{s}$ ame cluster, the cluster $\mathrm{c}$ ontaining web pages $\mathrm{h}$ ave $\mathrm{t}$ he $\mathrm{s}$ imilarity with respect to topic of the session. The clustering a lgorithms considered are $\mathrm{K}$-means and $\mathrm{K}$-mediods, $\mathrm{K}$ is determined by HITS algorithm. Finally, the predicted web pages are stored in form of cellular au tomata to make the s ystem more me mory efficient [12].

$\mathrm{Su}$ et al. (2000) s tudied the rapid development of internet has resulted in more and more multimedia in web content. The author studied due to the limitation in the bandwidth and huge size of the multimedia data, users always suffer from long time waiting. The author describe that to predict the web object or page that the user most likely will view next while the user is viewing the current page, and pre-fetch the content. Then the perceived $n$ etwork 1 atency can b e s ignificantly reduced. $T$ he author introduced n-gram based model to utilize path profiles of us ers from ve ry large w eb log to predict the us ers' future requests. Model is based o n a s imple e xtension o f e xisting point-based models for such predictions, but results show that by sacrificing the applicability somewhat one can gain a great deal in pr ediction pr ecision. $\mathrm{T}$ he $\mathrm{r}$ esults $\mathrm{c}$ an potentially $\mathrm{b} \mathrm{e}$ applied to a wide range of a pplications on the web, including pre-fetching, enhancement of recommendation systems as well as web ca ching $\mathrm{p}$ olicies. $\mathrm{T}$ he experiments $\mathrm{b}$ ased $\mathrm{o} \mathrm{n} \mathrm{t}$ hree realistic web logs have proved the effectiveness of the proposed scheme [13].

Zukerman et a l. (2009) used Artificial Intelligence-related techniques to pr edict user $\mathrm{r}$ equests. T he author i mplement a learning a lgorithm such a s some variation of $\mathrm{M}$ arkov chains and use a previous access $\log$ in order to train it. This approach also relies on tracking user patterns. Furthermore, it does not handle newly introduced pages, or old pages that have changed substantially. $\mathrm{T}$ his approach also $\mathrm{r}$ equires a $\mathrm{r}$ ather long sequence of clicks from a u ser to learn his/her access patterns [14].

Safronov et al. (2010) introduced the Page Rank based prefetching technique which is a server-side approach and uses the information about the link structure of the pages and the current and $p$ ast user accesses to $d$ rive pre-fetching. The a pproach is effective for access to w eb page c lusters, is computationally efficient and scalable, and can immediately sense and react to changes in the link s tructure of w eb p ages. F urthermore, the underlying a lgorithm u ses r elatively s imple matrix operations and is easily $\mathrm{p}$ arallelizable, making it s uitable f or $\mathrm{c}$ lustered server environments [15].

Padmanabhan et al. (1995) investigated ways of optimizing retrieval latency. $\mathrm{W}$ eb ca ching has been $\mathrm{r}$ ecognized as an effective solution to minimize user access latency. A method of called pre-fetching introduced in which clients in collaboration with server pre-fetch web page that the user is likely to access soon, $v$ iewing the cu rrently $d$ isplayed page 1 . The benefit of pre-fetching is to provide low retrieval latency for users, which can be explained as high hit ratio. This approach reduces web latency $b$ y $p$ re-fetching be tween caching, $p$ roxies, and browsers. Web pre-fetching has involved the important issue of log file processing and the determination of user transactions (sessions). It provides va rious data mining a lgorithms for the path traversal patterns and how to efficiently mine the access patterns from the web logs [16].

\section{PROPOSED MODEL}

Web page is the integration of the different web page contains frames, graphics and other information. User cache is used in this model to cache the frequently ac cessed web page. In the proposed m odel, w e r equest $t$ he certain $w$ eb page from the server then server will send the URL of that web page to the predictor. After that predictor check the required specific web page, if it is present then predictor gives that web page to the server and server also give required page to the client to meet its r equirements. B ut the predictor while sending the page to the $\mathrm{s}$ erver (in case the predictor i $\mathrm{s}$ ot a ble $\mathrm{t}$ o ch eck the requested web page), also give the client's requested web page to $t$ he $u$ pdate e ngine $f$ or upd ate a nd a lteration of the data structure. $\mathrm{T}$ he use of $\mathrm{p}$ redictor in the process of web pa ge anticipation is that it uses the data structure for storing the web pages. [17] 


\subsection{FLOW CHART OF REQUIRED MODEL}

The following flow chart presents the required model and the requisite steps to implement the required Markov model, which in turn helps in web page anticipation. In the proposed model, first step is to give input with the help of preprocessing the web server $\log$ files, after which similar web sessions are allocated to ap propriate ca tegories. B y us ing clustering, w e de cide the number of clusters and among these clusters web sessions are partitioned. $\mathrm{T}$ he $\mathrm{p}$ rocess of $\mathrm{c}$ lustering gives us the $\mathrm{c}$ lustered data which is used for the Markov model approach.

When the Markov model is applied w e $\mathrm{h}$ ave $\mathrm{d}$ ecide the prediction algorithm and then apply the hidden Markov model in prediction algorithm. After th e $d$ etermination o $\mathrm{f}$ th $\mathrm{e}$ prediction a lgorithm $t$ he ne $x t w$ eb pa ge f or us er a ccess i s available as output.

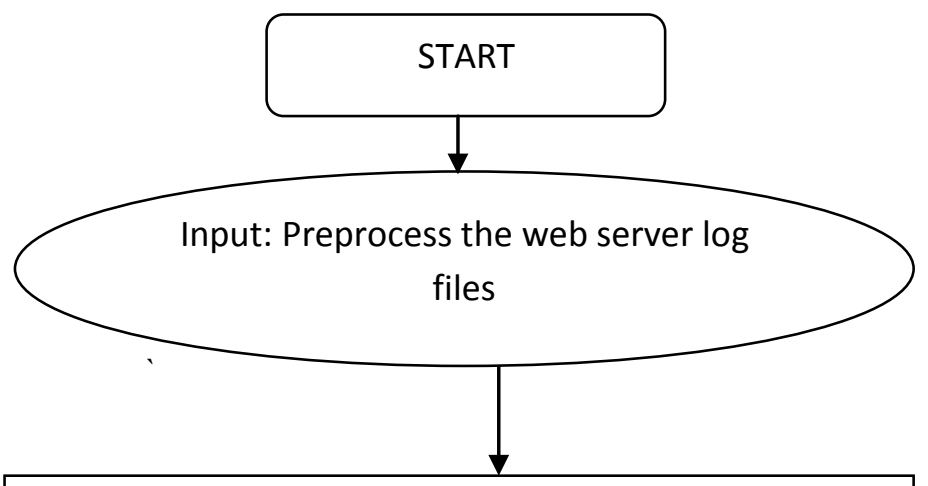

Decide on the number of clusters and partition the web sessions into clusters according to the chosen distance measure

Perform Markov model analysis on each of the clusters

Design the prediction algorithm and use Markov model

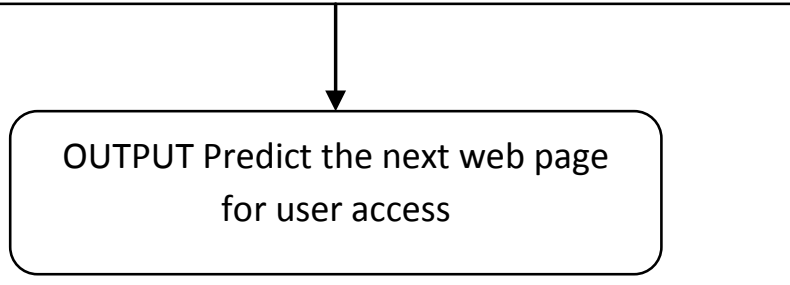

Figure2. flow chart for web page anticipation

\section{STEP1:}

web s erver 1 og files are preprocessed in a w ay s imilar web sessions are allocated to appropriate categories.

\section{STEP2:}

Convert $t$ he $\mathrm{w}$ eb pages i nto $\mathrm{n}$ umeric $\mathrm{f}$ orm an $\mathrm{d} \mathrm{s}$ tore $\mathrm{i} \mathrm{n}$ web.dat file and then determine the number of clusters and then division of web sessions into clusters is do ne us ing c lustering tool based on FCM algorithm on Matlab.

The FCM Algorithm uses the k-means clustering to choose the number of clusters $\mathrm{k}$. In this clusters centers U 1 to Uk are decided after that we could pick $\mathrm{k}$ data points and set cluster centers to these po ints or it could $r$ andomly a ssign po ints to clusters an $\mathrm{d} t$ ake m eans of $\mathrm{c}$ lusters. F or ea ch data p oint, we decide the cluster center it is closest to and assign the data point to this cluster.

After $t$ hat $w$ ith $t$ he he lp of findcluster $\mathrm{c}$ ommand on the command prompt on the matlab, the web.dat file is loaded on Matlab.

\section{STEP3:}

Perform M arkov m odel analysis o $\mathrm{n}$ e ach of the c lusters and then $m$ ake s quared Transition probability $m$ atrix and $s$ quared Emission Probability Matrix.

Making o f squared $\mathrm{T}$ ransition $\mathrm{P}$ robability Matrix (Rows $=$ Columns $=$ Total number of unique web pa ges $=9$ ) TRANSITION $(\mathrm{I}, \mathrm{J})$ is the probability of transition from state I to state $\mathrm{J}$

Making of $\mathrm{s}$ quared $\mathrm{E}$ mission $\mathrm{P}$ robability $\mathrm{M}$ atrix (Rows $=$ Columns $=$ Total num ber of uni que $\mathrm{w}$ eb pages $=9$ ) EMISSION $(K, L)$ is the probability of transition from state $\mathrm{K}$ to L.

\section{STEP4:}

Design $t$ he prediction a lgorithm a nd us e hi dden Markov Model approach. This algorithm gives us information about the next page with the help of user's currently accessed web page.

\section{ALGORITHM:-}

1. Set $\operatorname{Tr}=$ Transition square $\mathrm{m}$ atrix, $\operatorname{Tr}(\mathrm{I}, \mathrm{J})=$ Probability of transition from state I to state J. [Initializes $\mathrm{Tr}$ ]

2. Set $E=$ Transition square matrix, $\operatorname{Tr}(\mathrm{K}, \mathrm{L})=$ Probability of transition from state $\mathrm{K}$ to state $\mathrm{L}$. [Initialize $\mathrm{E}$ ]

3. Set seq $=$ sequence of user's accessed web page. [Initialize seq]

4. numStates $=$ number of states and size $=$ size of any column Tr. numStates $=$ size

5. L=length of seq

6. Repeat for count 1 To L

7. Repeat for state 1 to numStates

8. Set bestVal $=0$ [initialize bestVal]

9. Set bestPTR $=0$ [initialize bestPTR]

10. Repeat for inner 1 to numstaes

$$
\begin{aligned}
& \mathrm{Val}=\operatorname{Tr}[\text { innerstate }] \\
& \text { If } \mathrm{val}>\text { bestVal } \\
& \text { best } \mathrm{Val}=\mathrm{val} \\
& \text { bestPTR }=\text { val } \\
& \text { bestPTR }=\text { inner }
\end{aligned}
$$

[End of if structure]

[End of step 10 inner loop]

11. PTR $[$ state, count $]=$ bestPTR

12. $v[$ state $]=E[$ state, seq $[$ count $]]+$ bestval [End of 7 inner loop]

$13 . . \mathrm{vOld}=\mathrm{v}$ 


\section{4. $\mathrm{P}=\max [\mathrm{v}] \max$ is maximum value}

15. finalState $=\max [\mathrm{v}]$

16. currentState $[$ count $]=$ finalState

[End of step 6 outer loop]

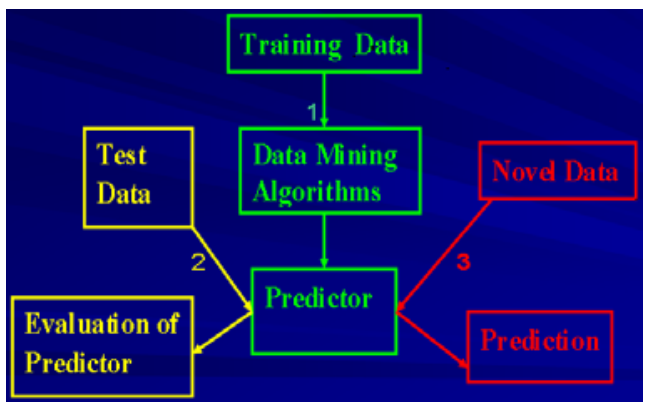

Figure 3. Prediction Architecture for training Data

\section{STEP 5:}

If we execute this prediction algorithm on the matlab then this w ill $g$ ive a next pr obable w ebpage f or us er's currently accesses webpage.

\section{RESULTS}

Merging w eb pages by w eb s ervices a ccording t ot heir function in $t$ urn reduces $t$ he nu mber o f uni que pa ges. The sessions $\mathrm{w}$ ere di vided $\mathrm{i}$ nto varying $\mathrm{c}$ lusters us ing $\mathrm{k}$-means algorithm a nd cosine function $m$ easure. For e ach cl uster, the categories were expanded back to their original form in the data set. This pr ocess i s pe rformed us ing a $\mathrm{s}$ imple program that seeks and displays the data related to ea ch ca tegory. Markov model implementation was carried out for the clusters. Markov model a ccuracy $\mathrm{w}$ as cal culated a ccordingly. Then, $\mathrm{u}$ sing the test $\mathrm{s}$ et, e ach $\mathrm{t}$ ransaction $\mathrm{w}$ as considered a s a ne $\mathrm{w}$ po int a nd distance measures were calculated in order to define the cluster that the po int be longs to. Markov model prediction a ccuracy was computed considering the transaction as a test set and only the $\mathrm{c}$ luster $\mathrm{t}$ hat the $\mathrm{t}$ ransaction be longs $\mathrm{t} \mathrm{o}$ a $\mathrm{s}$ a $\mathrm{t}$ raining $\mathrm{s}$ et. Prediction accuracy results were achieved using the maximum likelihood based on conditional probabilities.

All im plementations were $\mathrm{c}$ arried o ut using MATLAB. The Markov model accuracy was cal culated using a 10 -fold c ross validation. Results collected are user's currently ac cessed web page and $\mathrm{N}$ ext w eb page for u ser's cu rrently ac cessed web page. The reported accuracy is the how many Next web pages are u ser's actually ac cessed web p ages af ter u ser's currently accessed w eb $\mathrm{p}$ ages. Markov m odel accuracy u sing cl usters based on Cosine distance measures with $\mathrm{k}=4$.

All clustering runs have performed on a desktop PC with a Pentium IV Intel processor $r$ unning at $2 \mathrm{G} \mathrm{Hz}$ with $2 \mathrm{~GB}$ of RAM and 100 GB of hard disk memory. . The runtime of the kmeans algorithm, r egardless of the distance measure used, is equivalent to $O(n k l), n$ is the number of items, $k$ is the number of clusters a nd $l$ is the number of iterations $t$ aken $b$ y the algorithm to converge. For experiments, $n$ and $k$ are fixed, the algorithm has a linear time complexity in terms of the size of the da ta set. The k-means a lgorithm has a $O(k+n)$ space complexity. This is because it requires space to store the data matrix. It is feasible to s tore the data $\mathrm{m}$ atrix in a s econdary memory and then the space complexity will become $O(k)$. kmeans al gorithmi s more time an $\mathrm{d} s$ pace ef ficient $t$ han hierarchical c lustering a lgorithms with $O\left(n^{2} \quad\right.$ logn $)$ time complexity and $O\left(n^{2}\right)$ space complexity.

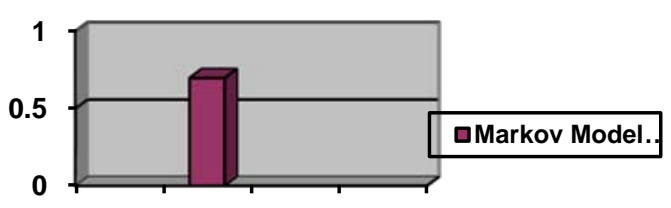

Figure 4. Markov Model accuracy

\section{CONCLUSION}

This $p$ aper $d$ escribes the $o$ verall $p$ rediction accuracy by grouping the data set sessions into clusters and reduces the web latency time. The web pages in the user sessions are segregated into categories according to Web services that are functionally meaningful. Then k-means clustering algorithm is implemented using the most appropriate number of clusters. The Prediction algorithm for the determination of us er's ne xt probable pa ge with the help of user's currently accessed page is applied on the number of clusters. The $\mathrm{r}$ esults gives us the a ccuracy of the next page access $\mathrm{p}$ rediction by $\mathrm{i}$ mplementing $\mathrm{t}$ he $\mathrm{k}$-means clustering algorithm on the data set decided previously. More accuracy describes most accurate web page predicted with the help of prediction algorithm implementation as user want next time. N ow us er do not ne ed to request the w eb page as us er wants, because it has been available before the time user want it $\mathrm{n}$ ext time. In this way we a re able to u tilize the minimum bandwidth of the user and the use of pre-fetching and clustering reduces the request made by user and reduces request time. The prediction a ccuracy achieved is an improvement o ver the problems o ccurred to the us er a t the time of accessing $\mathrm{w} \mathrm{eb}$ information on the Internet.

\section{FUTURE WORK}

This $\mathrm{p}$ aper in troduced th e Prediction a lgorithm $\mathrm{f}$ or automatically tr ansferring $\mathrm{W}$ eb pages. It $\mathrm{d}$ emonstrated that it can learn to closely reproduce human mappings, and it take a one step towards a powerful new paradigm for instance- web based de sign a nd opens up ex citing ar eas for $r$ esearch. A t present, the algorithm employs only about th irty simple visual and $\mathrm{s}$ emantic $\mathrm{f}$ eatures. E xpanding $\mathrm{t}$ his $\mathrm{s}$ et to include $\mathrm{m}$ ore complex and sophisticated properties, such as those ba sed on computer $\mathrm{v}$ ision, $\mathrm{w}$ ill lik ely im prove the robustness of $\mathrm{t}$ he machine le arning. A dditionally, th is im plementation cannot handle idiosyncrasies of modern HTML. Extending Prediction to these technologies remains future work.

\section{REFERENCES}

[1] Jia Yang, J. Zhang, and K. Beach, "A Survey of Web Caching Schemes for the Internet", ACM SIGCOMM, 2004.

[2] M. Liu , M. J unchang, a nd G . Zhimin, "Finding Shared Fragments in Large Collection of Web $\mathrm{P}$ ages for $\mathrm{F}$ ragmentbased Web Caching", Second IEEE International Symposium on Network Computing and Applications (NCA'06), 1998. 
[3] D. Kim, N. Adam, V. Alturi, M. Bieber, and Y. Yesha, "A Click Stream b ased Collaborative F iltering P ersonalization Model: Towards a b etter $\mathrm{p}$ erformance". WIDM' 04 , pages 8 8-95, 2004.

[4] W. Vakali, S. Yu, and D. Cai, "Improving ps eudo- relevance Feedback i n W eb I nformation R etrieval using W eb Page Segmentation", I $\mathrm{n}$ P roceedings of $\mathrm{t}$ he Twelfth I nternational World W ide W eb C onference, W WW2003, pp. 11-18, Budapest, Hungary, May 20-24, 2003.

R. Sarukkai, "Link P rediction a nd $\mathrm{P}$ ath Analysis u sing Markov C hains", 9th I nternational W WW C onference, Amsterdam, pages 377-386, 2000.

[6] Faten K halil, "Integrating Markov Model with Clustering for Predicting W eb Page A ccesses", W eb de velopment a nd mining, 2007.

[7] Mukund Deshpande, "Selective Markov Models for Predicting Web-Page A ccesses", IEEE/WIC/ACM I nternational Conference on Web Intelligence, 2001.

[8] Polyxeni Zacharouli, "Web Page Rank Prediction with PCA and EM Clustering", Web Engineering and Web mining, 2009.

[9] Myra Spiliopoulou, "A D ata Miner a nalyzing the Navigational Behaviour of Web Users", Data Mining, 1999
.[10] Debajyoti M ukhopadhyay, "Hybrid Web P age P rediction Model $f$ or Predicting U ser's Next A ccess", Information Technology Journal, 9: 774-781, 2011

.[11] Ranjitha Kumar, "Bricolage: A Structured-Prediction Algorithm for Example-Based Web Design", Web development and Data mining, 2011.

[12] Ruma D utta, “Clustering-based web pa ge p rediction”, Int. J . Knowledge and Web Intelligence, Vol. 2, No. 4, 2011.

[13] Zhong Su, "A Prediction System for Multimedia Pre-fetching in Internet", Microsoft Research China in Beijing, 2000.

[14] Zukerman, S. Y ang, J. Z hang a nd S . T sai, "An a utomatic Semantic- Segment Detection M ethod i $n$ the HTML Language", S ervices Computing, 2008. S CC a pos;08 IEEE International Conference on Volume 1, Issue , 2009.

[15] Victor Y. Safronov, "Strong Cache Consistency on World Wide Web", 3rd International Conference on Advanced Computer Theory and Engineering(ICACTE), 2010.

[16] Padmanabhan, "Using P redictive P refetching t o I mprove World Wide Web L atency", C OMPUTER COMMUNICATION, 1996.

[17] http://www.ijcmc.com/docs/papers/May2015/V415KJ0 3.pdf 